\title{
Analysis and Experiment of a Bio-inspired Flyable Micro Flapping Wing Rotor
}

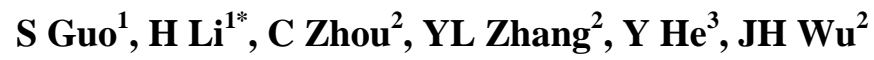 \\ ${ }^{1}$ Centre for Aeronautics, Aerospace, Cranfield University, Cranfield, Beds, UK \\ ${ }^{2}$ School of Transport Science and Engineering, Beihang University, Beijing, China \\ ${ }^{3}$ School of Aerospace Engineering, Beijing Institute of Technology, Beijing, China
}

\begin{abstract}
Inspired by insect flapping wings, a novel flapping wing rotor (FWR) has been developed for micro aerial vehicle (MAV) application. The FWR combines flapping with rotary kinematics of motions to achieve high agility and efficiency of flight. To demonstrate the feasibility of FWR flight and its potential MAV application, an extensive and comprehensive study has been performed. The study includes design, analysis, manufacture, experimental and flight test of a flyable micro FWR model of only $2.6 \mathrm{gm}$ weight. By experiment, the FWR kinematic motion and aerodynamic lift were measured using high speed camera and load cells. Within a range of input power, the difference between the measured aerodynamic force and the analytical results by a quasi-steady model was found to be within $3.1 \% \sim 15.7 \%$. It is noted that the FWR aeroelastic effect plays a significant role to obtain an ideal large angle of attack especially in up-stroke and enhance the FWR performance. Further analysis of the unsteady aerodynamic characteristics has been carried out based on the detailed airflow field of the FWR in a flapping cycle by CFD method. A successful vertical take-off and short hovering flight of the micro FWR model has been achieved for the first time in the research field. The flight test demonstrates the FWR feasibility and its unique feature of flight dynamics and stability for the first time. These characteristics have also been simulated by using ADAMS software interfaced with the aerodynamic model.
\end{abstract}

Keywords: Bioinspired flapping wing, flyable micro FWR, aeroelastic effect, flight simulation.

\section{Introduction}

Micro air vehicles (MAV) of high performance including the capability of vertical takeoff and landing (VTOL) and hovering capability can be operated in complex and risky environments including inside buildings. Two decade ago, the U.S. Defence Advanced Research Projects Agency (DARPA) launched a three-year MAV programme with the goal of creating a micro flyer for military surveillance and reconnaissance [1]. US Air Force Research Laboratory has the goal to develop a bird-sized MAV by 2015 and an insect-sized MAV by 2030. Motivated by the potential and growth demand of employing high performance micro air vehicles, various MAVs have been developed including fixed wing and rotorcraft configurations being used to carry out special missions. Inspired by the high agility of flying animals, research attention has also been attracted to studying bird-like and insect-like flapping wings [2-4].

\footnotetext{
${ }^{*}$ To whom any correspondence should be addressed - hao.li@cranfield.ac.uk
} 
In previous research, most of the attention was paid to the aerodynamic study of insect wing flapping in horizontal plane at very low Reynolds number $\left(\operatorname{Re} \sim 10^{3}\right)$ [5]. The subsequent study has revealed that the stall delay associated with attached leading edge vortex (LEV) plays a key role for high aerodynamic lift of insect flapping wings [6]. The LEV is stabilized by the spanwise flow which transports vorticity towards wingtip similar to a conventional delta wing, which produces majority of the high mean lift force (about 80\%) in a flapping cycle [7], while the rotational circulation and added mass effects contribute to the instantaneous lifting peaks at stroke reversals [8]. Experimental and numerical results also indicate that the vortex stretching could significantly delay the detachment of the LEV, even when the spanwise flow was weak [9]. Subsequent studies on rotary wing of small aspect ratio showed that the LEV could be stabilized by the centripetal and Coriolis accelerations at low Rossby number $\left(R o=\frac{R_{2}}{\bar{c}}\right.$, where $R_{2}$ is the radius of the second area moment, and $\bar{c}$ is the mean chord length) as the results of previous numerical and experimental studies $[10,11,12]$. In the same period, there were more investigations on the aerodynamic simulation of insect flapping wings $[13,14,15]$.

Because of the LEV attachment aerodynamic characteristics, the insect flapping wings are capable of operating at large angle of attack (AoA) to meet the requirement for high average lift coefficient. For the same reason however, the associated drag is also large in the same magnitude as the lift. As opposed to flapping wings, the conventional rotorcraft blade keeps operating at small AoA to prevent stall, but at high rotational speed to gain the required lifting force. Experimental studies have shown that at low $R e$ (100 14000), the rotary wing have higher aerodynamic efficiency in terms of lift production than flapping wing [10]. It was therefore suggested that the rotary wing may be superior for insect-sized MAV design in terms of energy efficiency [16]. However, the above findings are limited to the conventional rotary wing and the prescribed insect-like flapping wing kinematics.

In order to improve the aerodynamic efficiency of flapping wings, a bioinspired novel flapping wing rotor (FWR) was proposed earlier [17]. The FWR combines two types of motion, i.e. the insect-like flapping motion and man-made rotary motion. Driven by a vertical oscillating force to generate a prescribed primary flapping motion, the FWR produces lift and thrust forces simultaneously. The thrust propels the FWR to rotate in its horizontal plane. Since the FWR rotation is in a self-propelled passive mode, it keeps in a balanced equilibrium status of thrust against drag. For the same reason, there is no actuating torque and hence there is no need for counter rotation torque input, as opposed to the conventional rotorcraft. This feature leads to a power saving and system simplicity. In addition, the horizontal air velocity of the rotating FWR enhances the aerodynamic lift. Furthermore, it also leads to a significant reduction of necessary pitch angle in the up-stroke comparing with the dragonfly wing, which makes the FWR kinematics of motion feasible and practical. The FWR adds new specie in the bioinspired flapping-wing field as an alternative option for MAV application. Since then, increasing efforts have been contributed to the study of FWR aerodynamic behaviours. Guo and Zhou et al studied the aerodynamic performance of FWR by experimental and numerical methods $[18,19]$. By effectively changing the pitch angle in the up-stroke and down-stroke, the FWR will achieve different status for lift and efficiency. Wu et al [20] performed further detailed study of the forces produced by FWR at Re $=350 \sim 9000$ using CFD method. Their results showed that the LEV formed on the wing of FWR stays attached throughout the flapping cycle, which provides lift enhancement similar to insect wings.

In a recent work by the authors [21,22], the optimal kinematics of motion for FWR was identified and the aerodynamic lift and efficiency were calculated and compared with the conventional rotary and insect-like flapping wings. The results showed that the FWR can produce significantly greater aerodynamic lift coefficient and power efficiency than the insect-like flapping wings. The rotary wing has greater power efficiency, but smaller lift 
coefficient than the FWR and flapping wings. The FWR offers a significantly broader range of combination of aerodynamic lift and power efficiency. The FWR kinematics thus takes advantages of both the insect-like flapping wing and the rotorcraft and offers an alternative design for MAV.

Based on extensive studies of the FWR by the first author and his research teams in the last 10 years, investigation has been continued into the design, analysis, manufacture and experiment of a flyable micro FWR MAV test model. Driven by a micro electric motor and using carbon/epoxy composite to build most of the components, the total weight of the FWR test model is achieved to be only $2.6 \mathrm{gm}$. In the experiment, the wing kinematics and aerodynamic lift of the FWR model were measured using high speed camera and load cell. A desirable FWR wing structure was realised after a series of design and experiment to achieve the desired large pitch angle in the up-stroke for the FWR test model. The aerodynamic analysis was carried out using CFD method together with a quasi-steady aerodynamic method which employs empirical coefficients that accounts for unsteady aerodynamic effects $[23,24]$. The comparison of the analytical and experimental results shows excellent agreement. Subsequently a vertical take-off and short flight test of the FWR model was successfully carried out. This is, to our knowledge, the first flight test of the FWR vehicle. To further reveal the free-body dynamics and stability of the FWR model, flight simulation was performed using ADAMS combined with the quasi-steady aerodynamic method.

\section{FWR model and analysis method}

\subsection{FWR coordinate and kinematics}

The coordinate system for the FWR model including the inertial frame $(x, y, z)$ and the wing-fixed frame $\left(\mathrm{x}_{\mathrm{w}}, \mathrm{y}_{\mathrm{w}}, \mathrm{z}_{\mathrm{w}}\right)$ is defined and illustrated in Fig.1. The FWR device is essentially mounted with two wings in axial symmetry although only the right wing is shown in Fig.1. The pair of wing root $o$ is attached to a lever mechanism at the top of the FWR body. The rotation, flapping, and pitch angles of the wing are denoted by $\psi, \phi$, and $\alpha$, respectively.

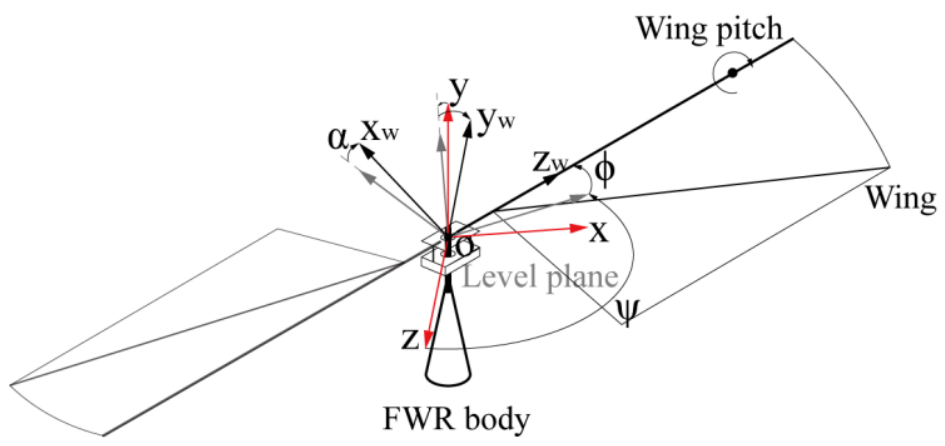

(a)

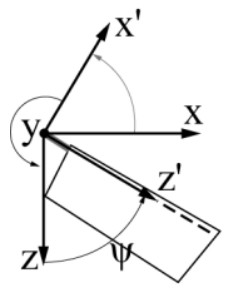

Rotation

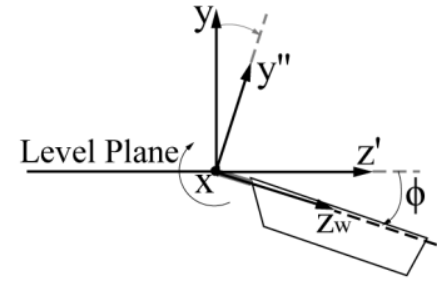

Flapping

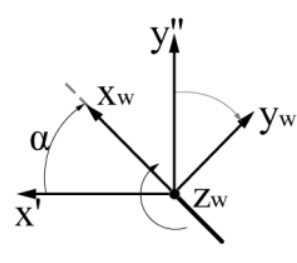

Pitching

(b)

Figure 1. (a) Coordinate system and rotation angle definition for the FWR model; (b) the top, back and side view of the Euler angles 
Based on the above definition, the angular velocity of the wing in the inertial frame can be obtained by the time derivatives of the three Euler angles as:

$$
\vec{\omega}_{\mathrm{i}}=\left[\begin{array}{c}
0 \\
\dot{\psi} \\
0
\end{array}\right]+\mathrm{R}(\psi)\left[\begin{array}{c}
\dot{\phi} \\
0 \\
0
\end{array}\right]+\mathrm{R}(\psi) \mathrm{R}(\phi)\left[\begin{array}{c}
0 \\
0 \\
\dot{\alpha}
\end{array}\right]
$$

where $\mathrm{R}(\psi)$ and $\mathrm{R}(\phi)$ are the rotation matrixes of the corresponding Euler angles. The angular acceleration in the inertial frame $\dot{\vec{\omega}}$ can be derived directly by differentiating the above equation. The angular velocity and acceleration of the wing in the wing-fixed frame are obtained by applying the following frame transformation:

$$
\mathrm{R}_{\mathrm{i} \rightarrow \mathrm{w}}=\mathrm{R}^{\mathrm{T}}(\alpha) \mathrm{R}^{\mathrm{T}}(\phi) \mathrm{R}^{\mathrm{T}}(\psi)
$$

The velocity and acceleration vector of a $2 \mathrm{D}$ wing chord at span-wise location $r$ in the wing-fixed frame are given by:

$$
\begin{gathered}
\overrightarrow{\mathrm{U}}(\mathrm{r})=\vec{\omega} \times \overrightarrow{\mathrm{r}}=\left[\begin{array}{lll}
\mathrm{u}_{\mathrm{x}} & \mathrm{u}_{\mathrm{y}} & 0
\end{array}\right]^{\mathrm{T}} \\
\dot{\overrightarrow{\mathrm{U}}}(\mathrm{r})=\dot{\vec{\omega}} \times \overrightarrow{\mathrm{r}}+\vec{\omega} \times(\vec{\omega} \times \overrightarrow{\mathrm{r}})=\left[\begin{array}{lll}
\dot{\mathrm{u}}_{\mathrm{x}} & \dot{\mathrm{u}}_{\mathrm{y}} & \dot{\mathrm{u}}_{\mathrm{z}}
\end{array}\right]^{\mathrm{T}}
\end{gathered}
$$

where $\vec{\omega}$ denote the rotation rate of the wing (in the wing-fixed frame); $\vec{U}(r)$ and $\overrightarrow{\dot{U}}(r)$ refers to the velocity and acceleration vector; $\mathrm{u}_{\mathrm{x}}, \mathrm{u}_{\mathrm{y}}$ and $\dot{\mathrm{u}}_{\mathrm{x}}, \dot{\mathrm{u}}_{\mathrm{y}}, \dot{\mathrm{u}}_{\mathrm{z}}$ are used to indicate the corresponding velocity and acceleration components. Based on the kinematic parameters, the effective angle of attack (AoA) of the wing at any instantaneous time can be determined by inversing the trigonometric function of the velocity ratio:

$$
\alpha_{\mathrm{e}}=\arctan \left(\frac{\mathrm{u}_{\mathrm{y}}}{\mathrm{u}_{\mathrm{x}}}\right)
$$

The kinematics of the wing is defined by three elementary motions: rotation, flapping and pitch, each corresponds to an Euler angle described above. The rotation of the wing is passively induced by aerodynamic force and the rotation speed at equilibrium is a constant number $\psi_{0}$. The wing flaps up and down passes through the level plane while pitches at the same time. The flapping angle and frequency are denoted by $\Phi$ and $f$. The pitch angles of the wing at mid-upstroke and mid-downstroke are denoted as $\alpha_{\mathrm{u}}$ and $\alpha_{\mathrm{d}}$, respectively. Fig.2 illustrates a typical FWR kinematics (simple harmonic motion) with a flapping amplitude $d$ and asymmetric AoA in up-stroke and down-stroke.

The above kinematic parameters in a series of flapping cycles for the experimental cases are obtained using discrete image processing method. The resulting wing motions are presented in section 3 .

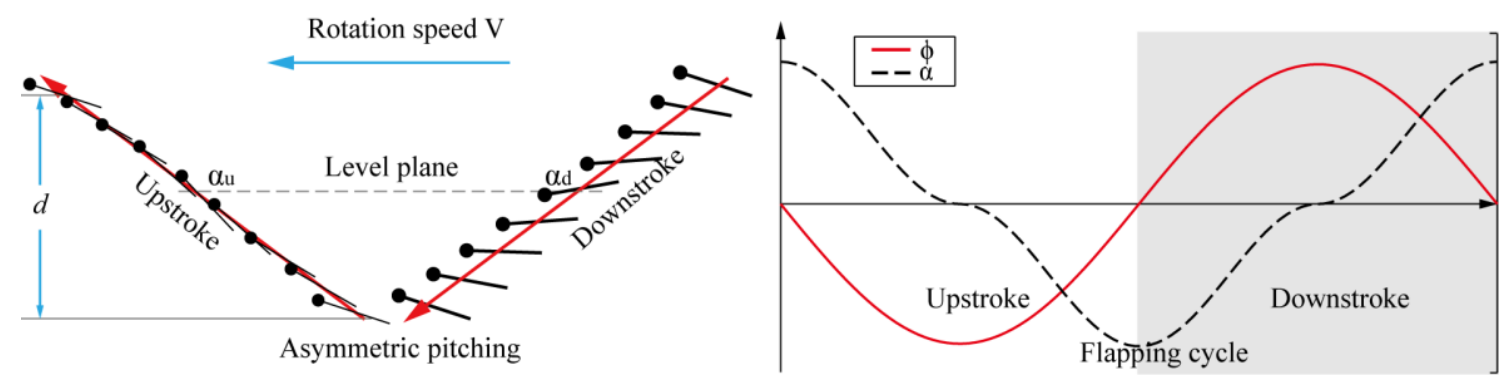

Figure 2. The typical kinematics of motion pattern of the FWR wing

\subsection{Quasi-steady aerodynamic model}


The flow around the periodically flapping wing is unsteady, which characterises strong growth and shedding of vortices from the leading and trailing edges and makes the aerodynamic forces difficult to be predicted from classical inviscid theory. Prior investigations of insect flapping wings at low Re measured a fluid force generation well beyond the steady-state value. However, extensive experimental and numerical studies have shown that the quasi-steady (QS) aerodynamic model by employing empirical forces coefficients can be used to model the instantaneous forces on flapping wings with high accuracy $[8,14]$.

In this model, the shape of the FWR wing is designed 'parametrically' which resembles a real insect wing according to the data measured by previous investigations [8]. The measurement for the wing geometry is illustrated in Fig.3. The aspect ratio (AR) of the wing model set in the present study is 3.3 since the recorded AR of typical insect wing is within $3 \sim 5$. The radial distribution of wing area, described by the first, second and third radius of dimensionless moments of wing area $\hat{\mathrm{r}}_{1}, \hat{\mathrm{r}}_{2}$ and $\hat{\mathrm{r}}_{3}$ (defined as: $\hat{\mathrm{r}}_{\mathrm{k}}=\left(2 \frac{\int_{0}^{\mathrm{R}} \mathrm{cr} \mathrm{r}_{\mathrm{dr}}}{\mathrm{SR}^{\mathrm{k}}}\right)^{\frac{1}{\mathrm{k}}}$, where $\mathrm{c}$ is the local chord length, $\mathrm{S}$ is the area of the wing pair, and $\mathrm{R}$ is the wingspan) is within the intervals $0.45 \sim 0.55,0.5 \sim 0.6$ and $0.55 \sim 0.65$, respectively [8].

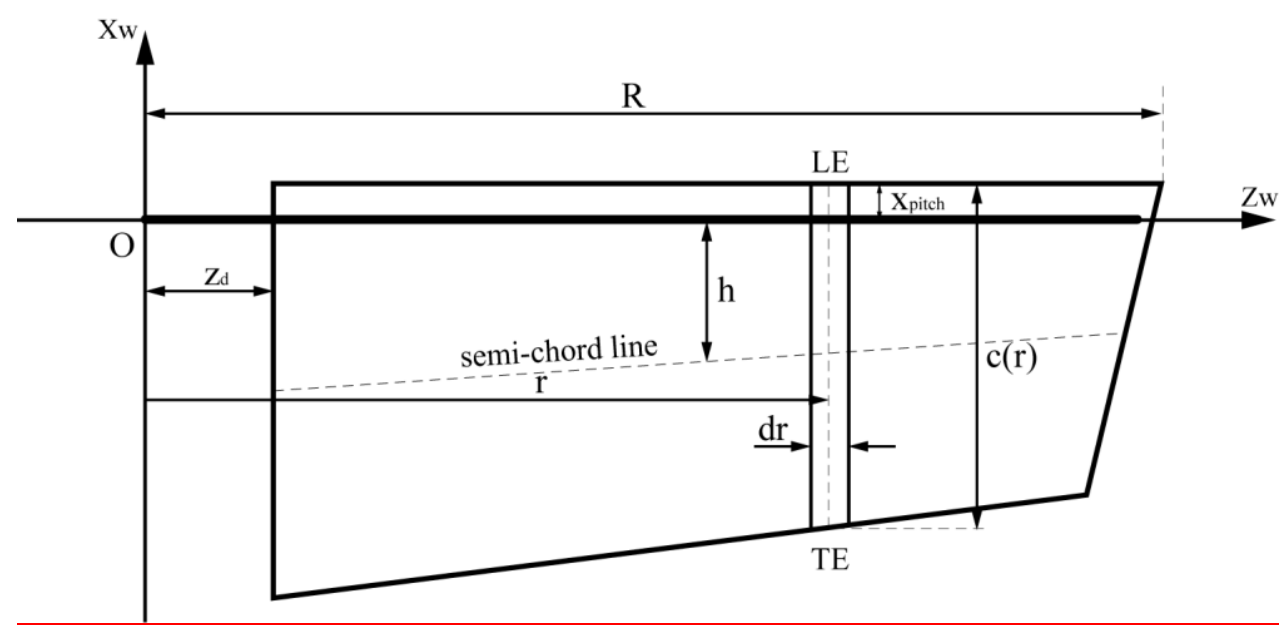

Figure 3. Wing geometric parameter definition of the FWR

The wing is of trapezoid shape, which has semi-span $\mathrm{R}$, and a root-cut at $\mathrm{z}$. The pitching axis of the wing $\left(\mathrm{z}_{\mathrm{w}}\right)$ is located near the leading edge; a 2D strip of the wing located at $r$ with width $\mathrm{dr}$ and local chord length $\mathrm{c}$ is shown in the figure. $\mathrm{h}$ is the semi-chord coordinate that can be transformed into a dimensionless form: $\hat{h}=\frac{h}{c}$ to measure the location of the pitching axis. It is taken as $\hat{h}=0.25$ in our calculation to match with observed data of insect wings $[18,22]$. The aerodynamic forces acting on a $2 \mathrm{D}$ wing strip follow by the equations:

$$
\begin{gathered}
\mathrm{d} F_{\mathrm{x}}(r)=\left\{\frac{1}{2} \rho|\vec{U}(r)|^{2} C_{\mathrm{H}}\left(\alpha_{\mathrm{e}}\right) c+\left(\lambda_{\mathrm{y}} u_{\mathrm{y}} \omega_{\mathrm{z}}-\lambda_{\mathrm{y} \omega} \omega_{\mathrm{z}}^{2}\right)\right\} \mathrm{d} r \\
\mathrm{~d} F_{\mathrm{y}}(r)=\left\{\frac{1}{2} \rho|\vec{U}(r)|^{2} C_{\mathrm{V}}\left(\alpha_{\mathrm{e}}\right) c+C_{\mathrm{rot}} \rho|\vec{U}(r)| \omega_{\mathrm{z}} c^{2}+\left(-\lambda_{\mathrm{y}} \dot{u}_{\mathrm{y}}+\lambda_{\mathrm{y} \omega} \dot{\omega}_{\mathrm{z}}\right)\right\} \mathrm{d} r
\end{gathered}
$$

where | I indicates the Euclidean norm; $\rho$ is the air density; $\alpha_{\mathrm{e}}$ is effective AoA of the wing obtained by equation (4); $\omega_{z}$ and $\dot{\omega}_{z}$ are the pitching rate and acceleration; $u_{y}$ and $\dot{u}_{y}$ are translational velocity and acceleration perpendicular to the wing chord; $\lambda_{y}$ and $\lambda_{y \omega}$ are the added mass force coefficients, which are given as $\lambda_{y}=\frac{\pi}{4} \rho c^{2}$ and $\lambda_{y \omega}=\frac{\pi}{4} \rho \hat{h} c^{3}$. The coefficients $C_{V}\left(\alpha_{e}\right), C_{H}\left(\alpha_{e}\right)$ and $C_{R O T}$ are the empirical coefficients for translational force and rotational force, respectively. 
The pitching moment of the 2D wing strip $d \tau_{z}$ is obtained by summing the translational moment $d \tau_{q s}$, the aerodynamic damping moment [25,26] $d \tau_{r d}$ and that due to added mass force $d \tau_{a m}$ :

$$
\mathrm{d} \tau_{\mathrm{w}}(r)=\mathrm{d} \tau_{\mathrm{qs}}+\mathrm{d} \tau_{\mathrm{rd}}+\mathrm{d} \tau_{\mathrm{am}}
$$

These terms are calculated, respectively, by the following equations:

$$
\begin{gathered}
\mathrm{d} \tau_{\mathrm{qs}}=-\frac{1}{2} \rho|\vec{U}(r)|^{2} C_{\mathrm{V}}\left(\alpha_{\mathrm{e}}\right) \hat{x}_{\mathrm{cp}} c^{2} \mathrm{~d} r \\
\mathrm{~d} \tau_{\mathrm{rd}}=-\frac{1}{2} \rho \omega_{\mathrm{z}}\left|\omega_{\mathrm{z}}\right| C_{\mathrm{rd}} \hat{x}_{\mathrm{rd}} c^{4} \mathrm{~d} r \\
\mathrm{~d} \tau_{\mathrm{am}}=\left[\lambda_{\mathrm{y}} u_{\mathrm{x}} u_{\mathrm{y}}-\lambda_{\mathrm{y} \omega}\left(\dot{u}_{\mathrm{y}}+u_{\mathrm{x}} \omega_{\mathrm{z}}\right)+\lambda_{\omega} \dot{\omega}_{\mathrm{z}}\right] \mathrm{d} r
\end{gathered}
$$

where $C_{r d}$ is the damping induced pitching moment coefficient; $\hat{x}_{c p}$ is the dimensionless centre of pressure; $\hat{x}_{r d}$ is the dimensionless location of the aerodynamic damping force. The added mass induced pitching moment coefficient $\lambda_{\omega}$ is given as [27]:

$$
\lambda_{\omega}=\left(\frac{\pi}{4} \hat{h}^{2}+\frac{\pi}{128}\right) \rho c^{4}
$$

The details of the empirical coefficients $C_{V}\left(\alpha_{e}\right), C_{H}\left(\alpha_{e}\right), C_{R O T}$ and $C_{r d}, \hat{x}_{c p}, \hat{x}_{r d}$ are presented in the previous study [8,14]. The 2D aerodynamic forces and pitching moment for each wing strip are integrated along the wing-span to obtain the 3D aerodynamic forces and pitching moment acting on the wing.

The vertical lift and rotational moment (moment along y-axis, see Fig.1) coefficients of the wing are defined by:

$$
\begin{aligned}
C_{L} & =\frac{L}{0.5 \rho U_{t}^{2} S} \\
C_{M} & =\frac{M}{0.5 \rho U_{t}^{2} S \bar{c}}
\end{aligned}
$$

where $U_{t}$ is the mean flapping velocity of the wingtip; $\bar{c}$ is the mean chord length; $L$ indicates the instantaneous lift and $M$ the corresponding rotational moment; $S$ is the wing area. The time averaged values of the lift and moment coefficients $\bar{C}_{L}$ and $\bar{C}_{M}$ are obtained similarly by taking the mean lift force $(\bar{L})$ and rotational moment $(\bar{M})$ over a flapping cycle in equation (9).

The quasi-steady aerodynamic model is validated by the CFD method with very good agreement of the results. The maximum difference of the QS model in lift and rotational moment coefficients are less than $12 \%$ and $15 \%$ respectively compared with 3D CFD results for a range of kinematic parameters. The details of the model validation and comparison are provided in the previous study [21].

\section{A Flyable FWR model and experiment}

\subsection{The FWR model design and manufacture}

The FWR model configuration is designed as shown in Fig. 4(a). A DC motor is mounted on the body frame made of carbon/epoxy beams. The rotational output of the motor is transformed into an up and down motion of a shaft through a crank-linkage mechanism. The top end of the shaft is connected through a bearing to a horizontal elastic plate. The pair of wing leading edge (LE) beam is connected at the root to each end of the elastic plate and supported by a U-frame through ball joints. The wings were set at a fixed initial geometric angle of attack $\left(\mathrm{AoA}=15^{\circ}\right)$. The three components (wing beam, U-frame and elastic plate) form an elastic lever mechanism that transmits the vertical action of the shaft to a flapping 
motion of the wings. The mechanism is able to rotate freely around the shaft together with the wings. Based on the design, a physical FWR model was built as shown in Fig. 4(b).

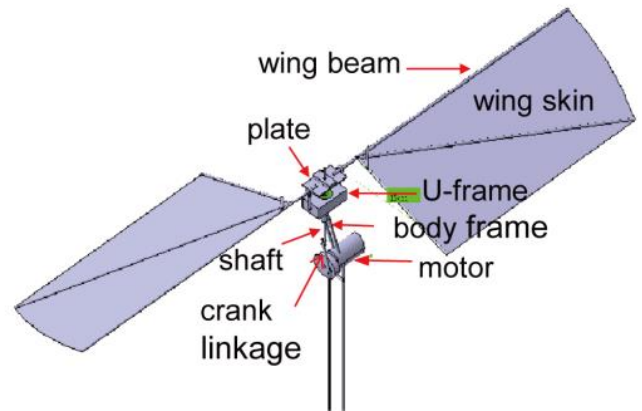

(a)

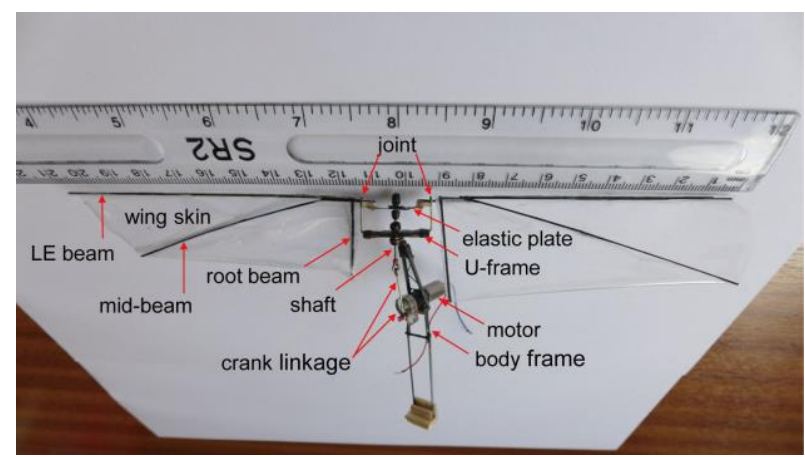

(b)

Figure 4. (a) FWR configuration and main components; (b) physical model

The dimensions and weight of the primary components of the FWR model are listed in Table 1. Further details of the wing configuration are shown in Fig.5.

Table 1. Dimensions and weight of the FWR model primary components

\begin{tabular}{|c|c|c|c|c|c|}
\hline FWR component & Wing & Body frame & Motor & Crank linkage & Others \\
\hline Dimension (mm) & $105 \times 33$ (single) & $75 \times 14 \times 7$ & $15 \times 6$ (radius) & 21 & - \\
\hline Weight (gm) & 0.28 (two) & 0.85 & 1.2 & 0.25 & 0.02 \\
\hline
\end{tabular}

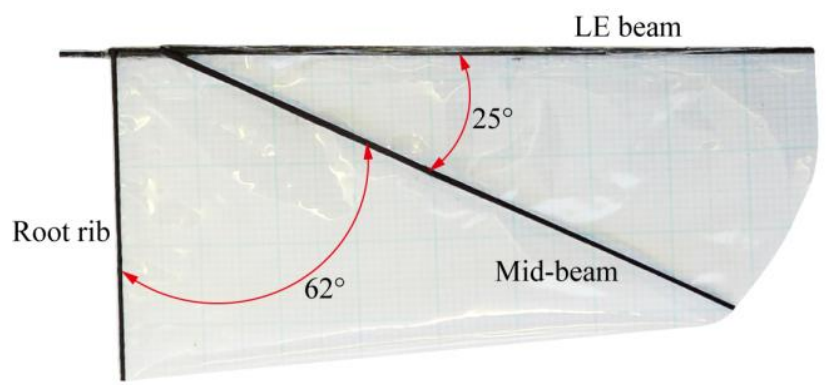

Figure 5. FWR model wing structure layout

The commercially available motor (ZWPD006006-700) integrated with a gear box was selected. Its standard operational rotation speed is $1200 \mathrm{rpm}$ with input voltage $3 \mathrm{~V}$ and current $25 \mathrm{~mA}$, but varies in a range of 500 1500 rpm with input voltage $1.5 \sim 4.5 \mathrm{~V}$. In the experiment, the required electric power was supplied from a DC power unit.

In the design and manufacturing of the FWR model, the key challenge is for the wing to achieve an optimal kinematics of motion with a sufficient large $\alpha_{u}$ (AoA in up-stroke) and small $\alpha_{\mathrm{d}}$ (AoA in down-stroke). Since the actuated motion is in vertical direction only, the AoA of a flapping cycle varies in a passive manner that relies on the flexibility of the lever mechanism and the wing structure. Therefore, the elastic plate plays a key role of the flapping mechanism. It not only amplifies the wing flapping angle, but also allows a large twist of the wing due to aeroelastic effect during flapping motion.

In order to make the crank in line with the actuation shaft, the motor was mounted in a position where its center of gravity (CG) is eccentric from the FWR center line as shown in Fig.4. This arrangement results in the CG of the FWR model having $3.5 \mathrm{~mm}$ distance from the FWR center in z-direction (see Fig.1). 


\subsection{The FWR model experiment}

The FWR model was mounted on a load cell (force transducer, SINOSERA, CL-YB-8/5N in accuracy $\pm 0.2 \%$ ) to measure the instantaneous dynamic force in transverse direction; the force from the load cell was transmitted to a signal amplifier at a sampling rate of $3000 \mathrm{~Hz}$; the FWR kinematics of motion was captured using a high speed camera at a data rate of 1000 frame/s as illustrated in Fig. 6(a). The two types of data were transmitted simultaneously and recorded in the PC. The experiment setup is shown in Fig. 6(b).

The kinematics of motion of the FWR model was obtained by post-processing the images captured using the high speed camera. The flapping frequency $f$ and rotational speed $n$ is determined by counting the number of frames over 10 flapping cycles $N_{f}$ and rotational circles $N_{n}$ as: $f=(10 \times 1000) / N_{f}$ and $n=(10 \times 1000) / N_{n}$.

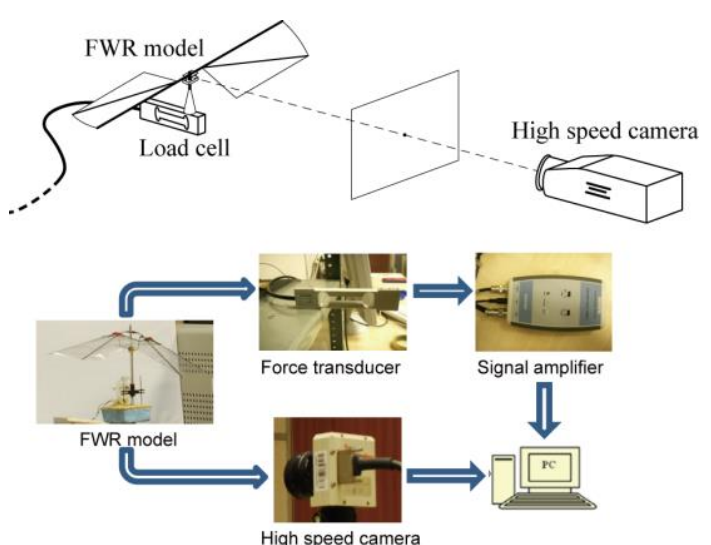

(a)

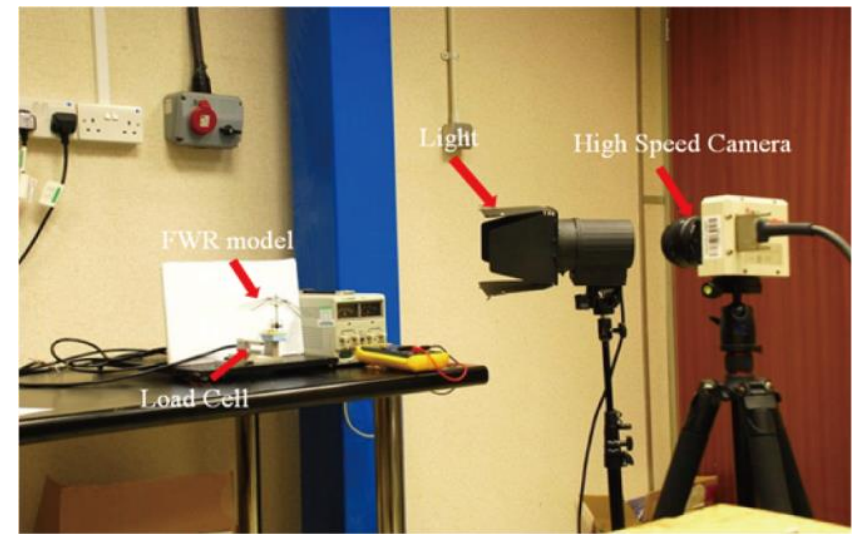

(b)

Figure 6. (a) The FWR measurement devices and (b) experiment setup

A series of FWR experiments was performed in a range of input voltages $2.5 \sim 4.5 \mathrm{v}$ with each measurement time lasting 10s. The instantaneous aerodynamic force was obtained by removing the symmetric periotic inertia force from the measured force data. The resulting FWR flapping frequency, maximum flapping angle (flapping amplitude), AoA, rotation speed and average aerodynamic lift were measured and summarised in Table 2 and as shown Fig. 7(a). In addition, the ratio of output lift $(\mathrm{N})$ to input power $(\mathrm{W})$ as a measure of the power efficiency and the Strouhal number $(\mathrm{St}=f d / V$, where $f$ is flapping frequency, $d$ is the flapping amplitude at wingtip and $V$ is the rotation velocity) is calculated and presented in Table 2 and Fig. 7(b).

Table 2. The FWR model results from input voltage in the range of $2.5 \sim 4.5 \mathrm{v}$

\begin{tabular}{ccccccc}
\hline Input voltage & $(\mathrm{v})$ & 2.50 & 3.0 & 3.50 & 4.0 & 4.5 \\
\hline Input power & $\mathrm{P}(\mathrm{W})$ & 0.13 & 0.18 & 0.23 & 0.28 & 0.34 \\
Max flapping angle & $\Phi($ deg. $)$ & 35.0 & 39.0 & 36.0 & 41.0 & 35.0 \\
AoA in mid-up-stroke & $\alpha_{\mathrm{u}}($ deg. $)$ & 38.0 & 40.0 & 39.0 & 40.0 & 41.0 \\
AoA in mid-down-stroke & $\alpha_{\mathrm{d}}(\mathrm{deg})$. & 7.00 & -4.0 & -10.0 & -18.0 & -22.0 \\
Flapping frequency & $f(\mathrm{~Hz})$ & 11.42 & 14.01 & 19.31 & 21.79 & 24.10 \\
Rotation speed & $\mathrm{n}(\mathrm{r} / \mathrm{s})$ & 2.78 & 4.57 & 6.21 & 7.81 & 8.93 \\
Average lift & $\mathrm{L}(\mathrm{mN})$ & 11.7 & 17.4 & 24.0 & 27.2 & 29.7 \\
Mean lift coefficient & $\overline{\mathrm{C}}_{\mathrm{L}}$ & 1.26 & 1.00 & 0.86 & 0.59 & 0.72 \\
Strouhal number & $\mathrm{St}$ & 0.39 & 0.32 & 0.31 & 0.31 & 0.26 \\
\hline
\end{tabular}




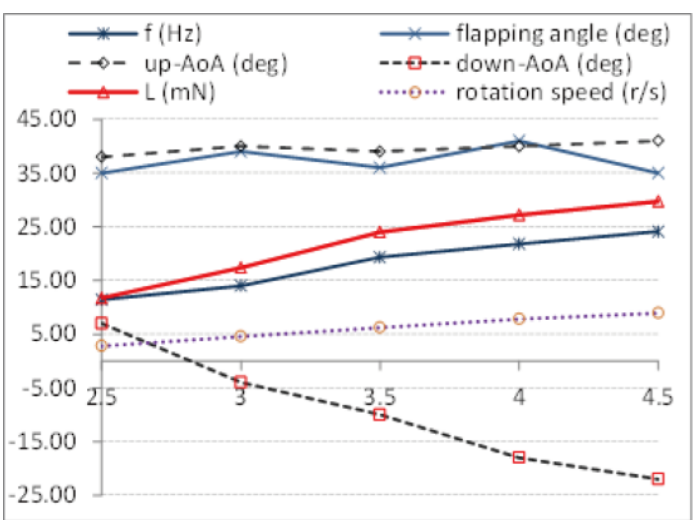

(a)

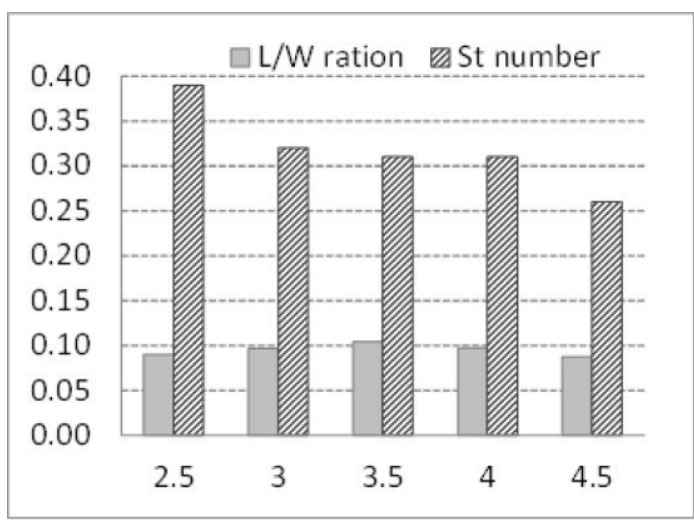

(b)

Figure 7. (a) The resulting FWR model flapping frequency, angle, geometric AoAs, mean lift and rotation speed; (b) lift to power ratio and $S t$ for input voltage $2.5 \sim 4.5 \mathrm{v}$

It is noted from the experimental results that the FWR flapping frequency, rotation speed and average lift increase almost linearly with the input power $(2.5 \mathrm{v} \sim 4.5 \mathrm{v})$. During the flapping motion, the wing AoA varies passively around the initial geometric $\mathrm{AoA}=15^{\circ}$ as a result of the aeroelastic interaction between the wing elastic, inertia and aerodynamic forces. When the input voltage increased from $2.5 \mathrm{v} \sim 4.5 \mathrm{v}$, the up-stroke $\alpha_{\mathrm{u}}$ increased from $38^{\circ} \sim 41^{\circ}$ corresponding to an aeroelastic twist angle of $23^{\circ} \sim 26^{\circ}\left(15^{\circ}+23^{\circ}=38^{\circ} \sim 15^{\circ}+26^{\circ}=41^{\circ}\right)$. In the down-stroke, the variation and magnitude of the aerodynamic force and (negative) pitching moment are significantly greater than the up-stroke. The pitching moment produced a large variation of (negative) aeroelastic twist angle from $-8^{\circ} \sim-37^{\circ}$ that resulted in the $\alpha_{\mathrm{d}}$ varied from $15^{\circ}-8^{\circ}=7^{\circ} \sim 15^{\circ}-37^{\circ}=-22^{\circ}$ for the input $2.5 \mathrm{v} \sim 4.5 \mathrm{v}$.

The kinematics of motion $\left(\alpha_{\mathrm{u}}=41^{\circ}, \alpha_{\mathrm{d}}=-22^{\circ}\right)$ at the maximum input $4.5 \mathrm{v}$ is close to the optimal solution $\left(\alpha_{\mathrm{u}}=42^{\circ}, \alpha_{\mathrm{d}}=-24^{\circ}\right)$ of maximum aerodynamic efficiency of a generic FWR [21]. It is also interesting to note that the $S t$ of the FWR falls in the range of optimal propelling efficiency $0.25 \sim 0.4$ as demonstrated by flying animals [28] over the input power range as shown in Table 2 and Fig. 7(b). Although the St in Table 2 are calculated at the wingtip, they remain the same for any section of the FWR.

Unlike the aerodynamic force, the mean lift coefficient of the FWR does not vary progressively with the input power. Although the lowest input $2.5 \mathrm{v}$ resulted in the highest $\bar{C}_{L}=1.26$ for the FWR model, the passive kinematics of motion $\left(\alpha_{\mathrm{u}}=38^{\circ}, \alpha_{\mathrm{d}}=7^{\circ}\right)$ is not close to the optimal solution shown in previous study $\left(\alpha_{\mathrm{u}}=18^{\circ}, \alpha_{\mathrm{d}}=-3^{\circ}\right.$ [21]). The results indicate that an optimal FWR kinematics of motion for maximum $\bar{C}_{L}$ requires a prescribed actuation rather than achieved by passive twist.

From Fig.7(b), it is noted that the power efficiency reached the maximum for the motor in optimal operation power $(3.5 \mathrm{v})$. The resulting lift force $L=24 \mathrm{mN}$ is only $2 \mathrm{mN}$ smaller than the required value to lift up the FWR model. This case is thus taken for further detailed study.

In order to measure the kinematics of motion of the model in this particular case $(3.5 \mathrm{v})$, the FWR images were captured at a series of discrete instantaneous moment and post-processed as shown in Fig. 8. The maximum flapping angle and AoA at the mid-up-stroke and middown-stroke was obtained from processing the measured data as shown in Fig. 9. 

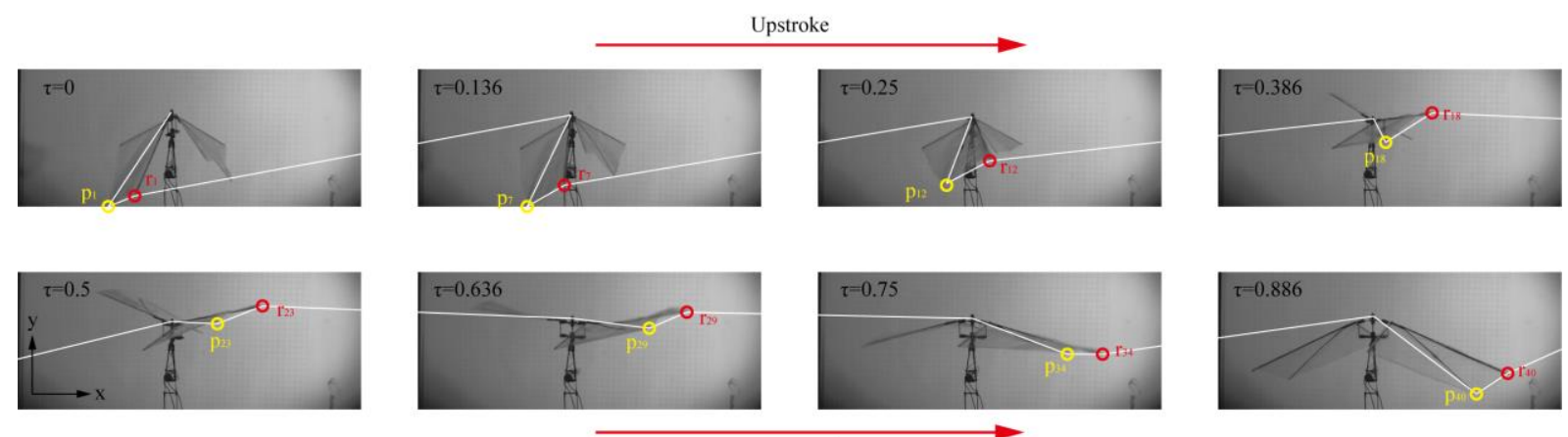

Figure 8. FWR image processing for 3.5v input voltage

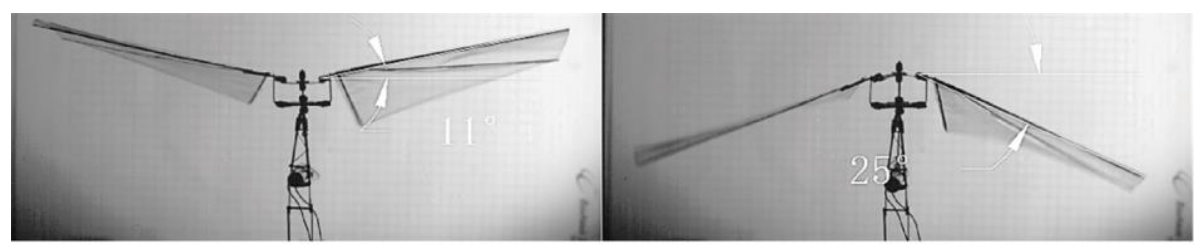

(a)

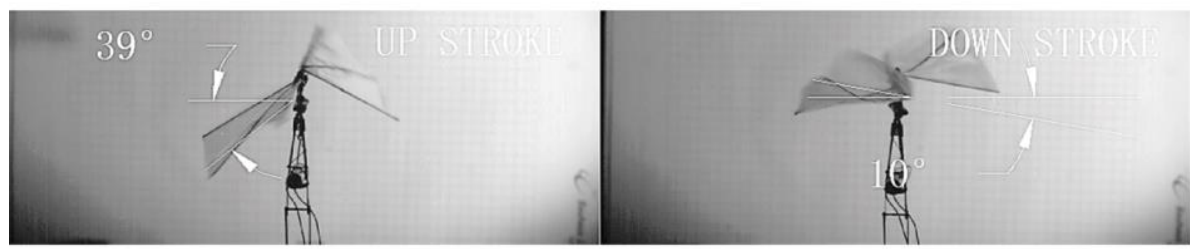

(b)

Figure 9. (a) Flapping angle at up most and lowest position; (b) AoA at mid-up-stroke $\left(\alpha_{\mathrm{u}}\right)$ and mid-down-stroke $\left(\alpha_{d}\right)$ for input $3.5 \mathrm{v}$

An analytical function of the FWR kinematics of motion was created by curve fitting the measured data ( $3.5 \mathrm{v}$ case) over a couple of flapping cycles using a Fourier trigonometric series. The processed FWR flapping angle and AoA results are shown in Fig.10. The velocities and accelerations of the wing motion were obtained by differentiating the displacement function in time.

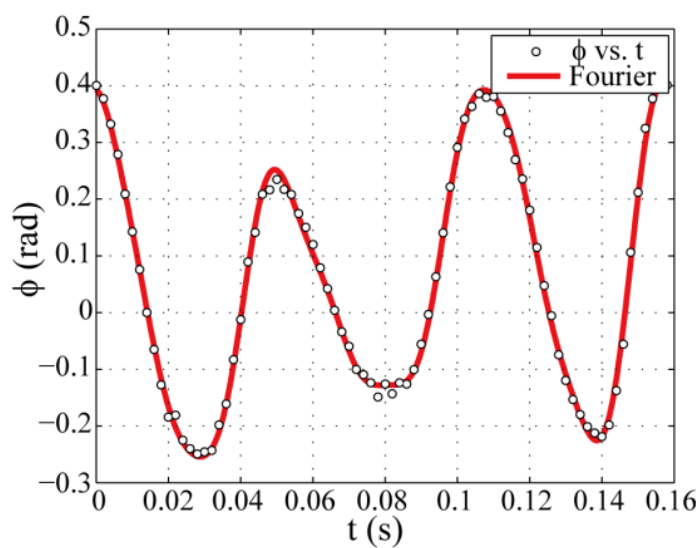

(a)

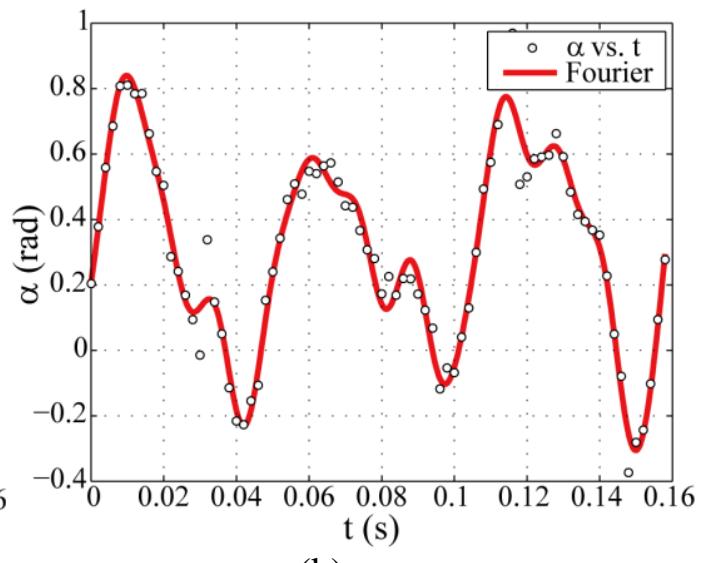

(b)

Figure 10. Curve fitting of the measured (a) flapping angle; (b) AoA for 3.5v case. White dots indicate measured kinematic data; red line indicates fitted function using Fourier series

\section{The FWR model theoretical, CFD and experimental results}




\subsection{Comparison of theoretical, CFD and experimental results}

According to the function created from the measured FWR kinematics of motion, the aerodynamic forces were calculated using CFD method and the quasi-steady (QS) aerodynamic model presented in section 2.2 [8,14,21]. The CFD simulation in the present study was carried out by solving the 3D incompressible unsteady Navier-Stokes equations of the flapping wing in assumed laminar flow. Details of the flow solver and the validation of our code can be found in the previous work [22]. Grid-independence of CFD simulations are performed with the grid node quantities $67 \times 81 \times 78$ (in normal, chordwise, and spanwise directions, respectively); the outer boundary is located 30 chords away from the wing surface and 15 chords away from the wing tip; 400 time steps per flapping cycle is used in the simulation.

In the CFD and QS models, the aeroelastic effect of the FWR wing has been partly taken into account by using the instantaneous AoA measured during the experiments as shown in Fig.9, Fig.10. The data is influenced by the wing twist deformation although the effect of wing shape change, i.e. spanwise bending and chordwise camber deformation has been ignored. The data provide reasonably good approximations since previous study shows that the wing shape change effect would not cause significant change of aerodynamic forces $<10 \%$ [29].

Corresponding to the input $3.5 \mathrm{v}$, the FWR motion was measured in terms of flapping angle $\Phi$ and AoA. According to the motion, the lift and drag coefficients were calculated using the two methods and shown over six flapping cycles in Fig. 11. The positive $\Phi$ indicates the wing flapping position below the horizontal plane; negative value above the plane.

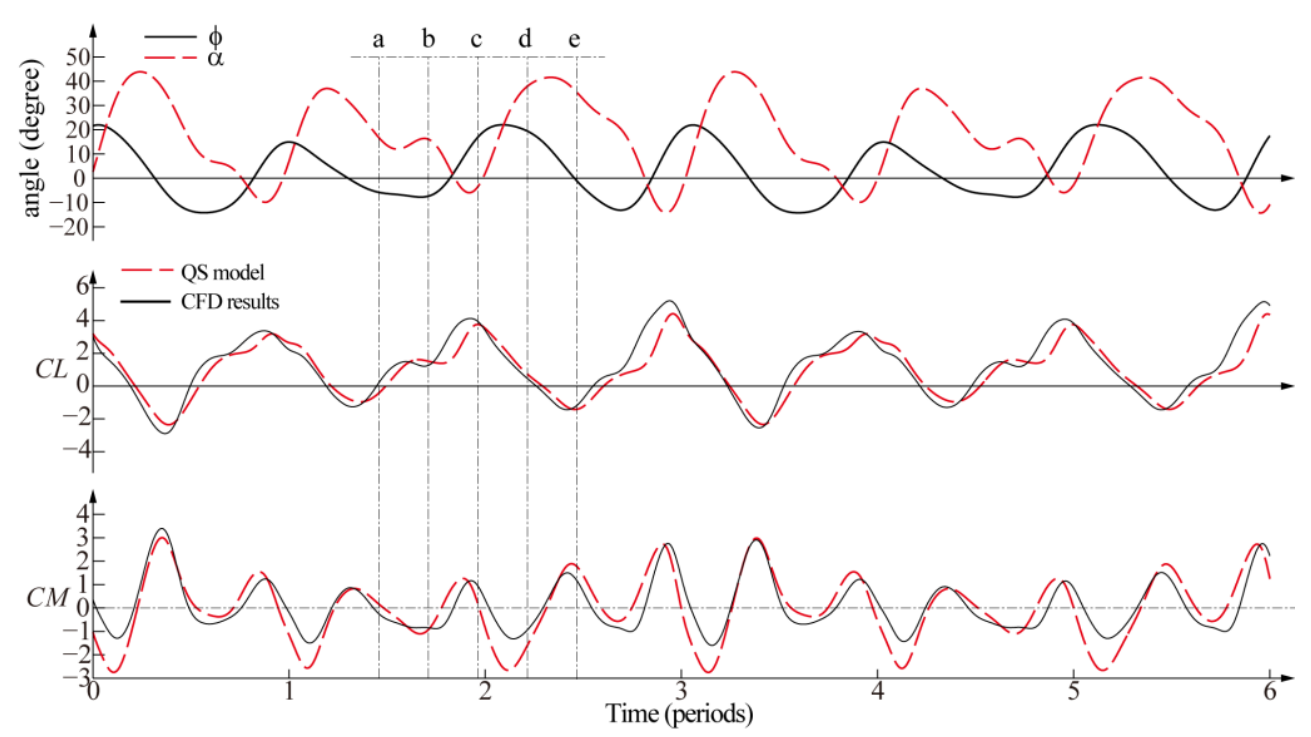

Figure 11. The FWR kinematics of motion, $C_{\mathrm{L}}$ and $C_{\mathrm{M}}$ using CFD and QS methods for 3.5v

The instantaneous $C_{\mathrm{L}}$ and $C_{\mathrm{M}}$ from both methods show excellent agreement. Based on the results, the average aerodynamic forces and mean lift coefficients over the six flapping cycles were calculated and compared with the experimental results as shown in Table 3 and Fig.12. In Fig.12, the error bands at each input voltage are obtained by choosing the maximum and minimum measured mean lift of different flapping cycles within the interval of measurements (10s in total).

Table 3. Comparison of analytical \& experimental results 


\begin{tabular}{cccccc}
\hline Input voltage (v) & 2.5 & 3.0 & 3.5 & 4.0 & 4.5 \\
\hline Measured Lift (mN) & 11.4 & 17.4 & 24.0 & 27.2 & 29.7 \\
QS-Method Lift (mN) & 9.6 & 17.2 & 24.8 & 28.3 & 32.0 \\
Experimental $\overline{\mathrm{C}}_{\mathrm{L}}$ & 1.33 & 1.35 & 0.98 & 0.87 & 0.78 \\
QS-Method $\overline{\mathrm{C}}_{\mathrm{L}}$ & 1.12 & 1.33 & 1.01 & 0.90 & 0.84 \\
CFD Lift $(\mathrm{mN})$ & - & - & 29.8 & - & - \\
CFD $\overline{\mathrm{C}}_{\mathrm{L}}$ & - & - & 1.21 & - & - \\
\hline
\end{tabular}

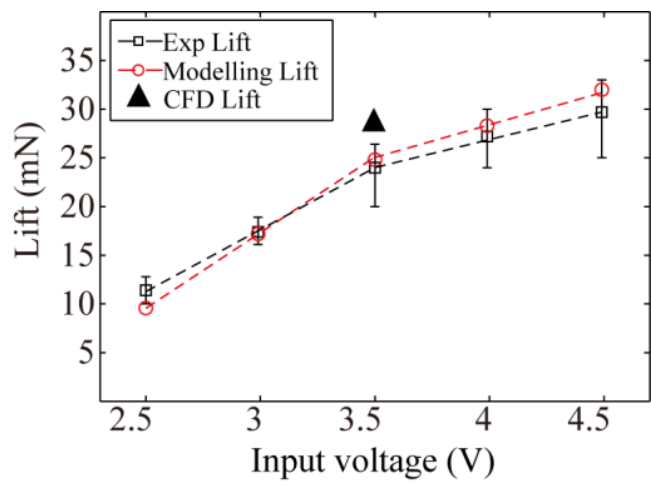

(a)

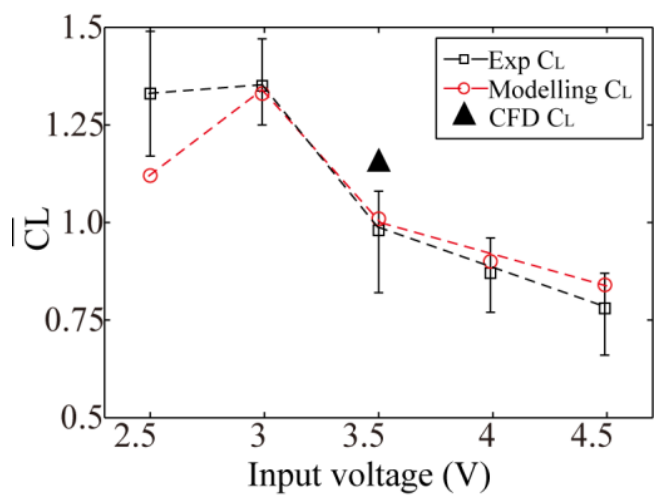

(b)

Figure 12. (a) The FWR average lift and (b) mean $\overline{\mathrm{C}}_{\mathrm{L}}$ from experiment and QS method for the range of input voltages $2.5 \mathrm{~V} \sim 4.5 \mathrm{v}$

The result comparison indicates that the mean lift coefficient and resulting force for the input voltage $3.5 \mathrm{v}$ were overestimated by CFD method. The difference between measured and analytical lift coefficients and forces is less than $3.4 \%$ when the input power is in the designed motor operation range around $3 \sim 3.5 \mathrm{v}$. For the input voltages $4 \mathrm{v} \sim 4.5 \mathrm{v}$ beyond the range, the difference is increased to $7.7 \%$. When the motor was under performed for low power input $2.5 \mathrm{v}$ however, the analytical lift was underestimated with a deviation of $15.8 \%$. This is mainly because the FWR AoA became significantly irregular due to passive aeroelastic effect particularly in the up-down stroke transition period near the maximum up flapping angle. This resulted in an increasing deviation for the curve-fitting and approximated function of the FWR kinematics of motion as shown in Fig.13. For more accurate analytical result, the curvefitting process should be improved. 


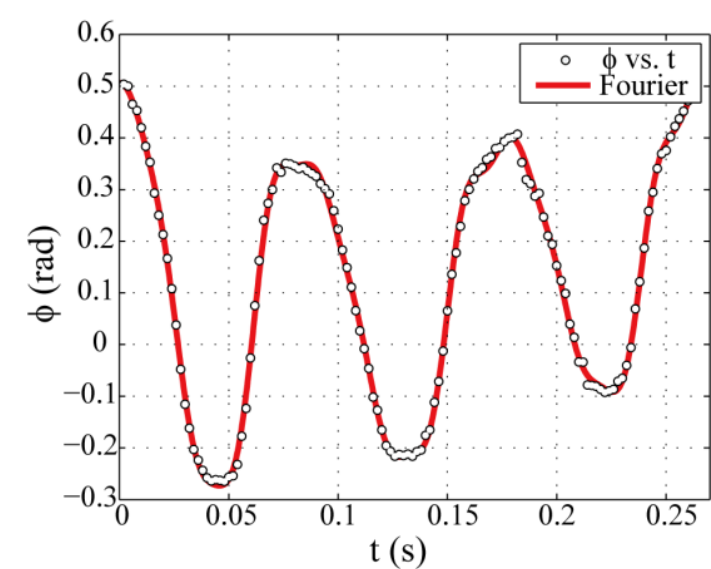

(a)

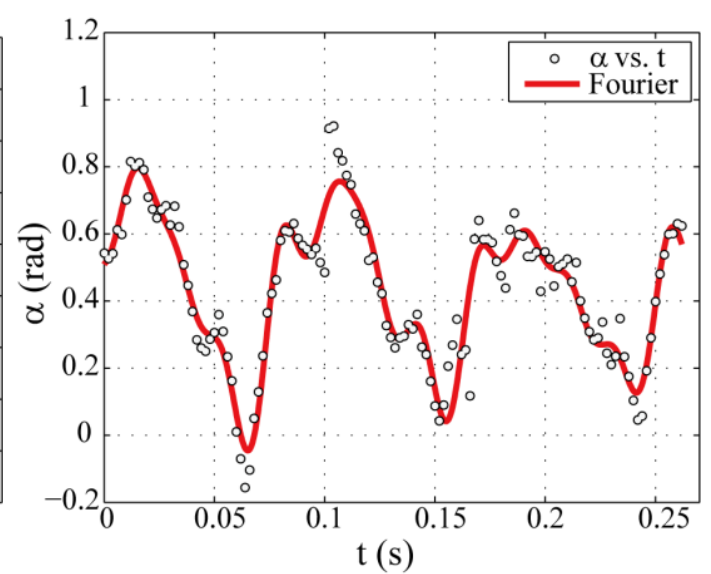

(b)

Figure 13. Curve fitting of the measured (a) flapping angle; (b) AoA for input 2.5v

\subsection{Analysis of CFD results}

In order to further study the aerodynamic details, the flow field obtained by the CFD method at five moments of time $(\mathrm{a} \sim \mathrm{e})$ over one of the flapping cycles as marked in Fig.11 are selected and presented in Fig.14. For this selected cycle, the maximum flapping angle $\Phi=31^{\circ}$ (lower $\phi=22^{\circ}$, upper $\phi=-9^{\circ}$ ) and wingtip displacement $d=0.057 \mathrm{~m}$. At the flapping frequency $19.3 \mathrm{~Hz}$, the average flapping velocity at wingtip is $U=2 f d=2.2 \mathrm{~m} / \mathrm{s}$. In the same time, the FWR rotation produced a forward (horizontal) velocity $V=3.9 \mathrm{~m} / \mathrm{s}$ (wingtip). It is noted that the ratio of $U / V$ equals to double of the $\mathrm{St}=f d / V$. The FWR motion changes the instantaneous effective AoA of the wing. The effect of the deflected velocity can be studied qualitatively by the mean deflection angle $\alpha_{q}=\operatorname{Tan}^{-1}(U / V)=+29.3^{\circ}$ in the down-stroke and $29.3^{\circ}$ in the up-stroke, which remains the same along the wing span (ignoring the chord-wise deformation). In this study, the flapping cycle is divided into four quarters period. The $1^{\text {st }}$ and $2^{\text {nd }}$ quarter corresponds to the upper half of the up-stroke and down-stroke respectively when the wing is above the horizontal $(x-z)$ plane $(y>0, \phi<0)$. The time-a and time-b as shown in Fig. 11 is in mid of the two quarters respectively. The $3^{\text {rd }}$ and $4^{\text {th }}$ quarter corresponds to the lower half of the down-stroke and up-stroke including time-c and time-d respectively when the wing is below the $\mathrm{x}-\mathrm{z}$ plane $(\mathrm{y}<0, \phi>0)$. The time-e is at the end of the $4^{\text {th }}$ quarter cycle as shown in Fig.11.
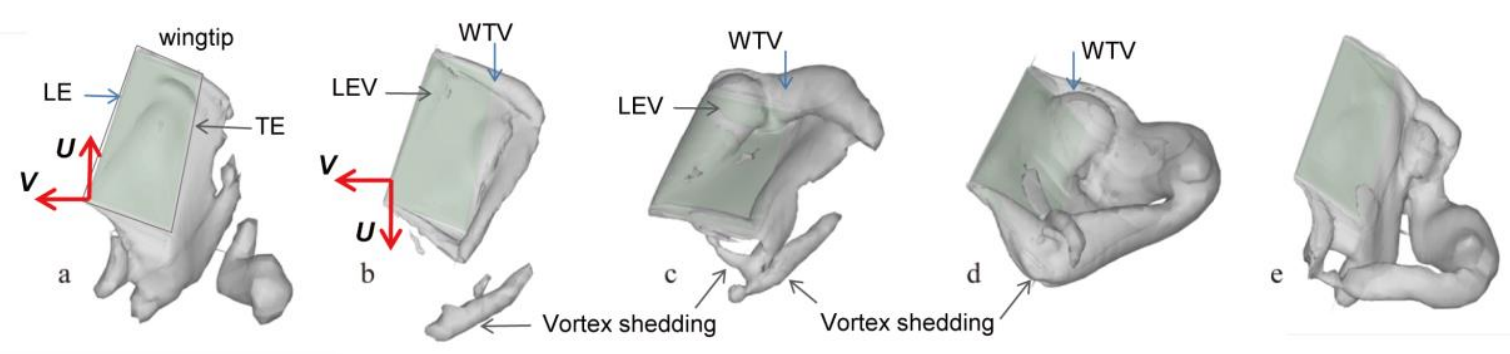

Figure 14. The flow field simulation by CFD at five time moments over a flapping cycle

At a time moment in phase-a, the wing was in the $2^{\text {nd }}$ half of up-stroke (mid of the $1^{\text {st }}$ quarter cycle) with geometric AoA $\alpha_{\mathrm{u}}=17^{\circ}$ as shown in Fig. 11 . The combined $\alpha_{\mathrm{u}}$ and quasisteady $\alpha_{\mathrm{q}}=-29.3^{\circ}$ resulted in an equivalent $\alpha_{e}=\alpha_{\mathrm{u}}+\alpha_{q}=17^{\circ}-29.3^{\circ}=-12.3^{\circ}$ of the FWR against the airflow. At this moment, the wing LE and upper surface is relatively clean, but the vortex 
was generated over the lower surface and shed off the TE as shown in Fig.14(a). Under this flow condition, the FWR produced a small lift coefficient $C_{\mathrm{L}}=0.15$ and rotational moment coefficient $C_{\mathrm{M}}=-0.25$ as shown in Fig. 11 .

At a time moment in phase-b, the FWR wing was accelerated in the first half of downstroke (mid of the $2^{\text {nd }}$ quarter cycle). Although the wing has completed the transition from upstroke to down-stroke, the geometric AoA $\alpha_{\mathrm{d}}=17^{\circ}$ was kept almost the same as time-a, and the resulting equivalent $\alpha_{\mathrm{e}}=\alpha_{\mathrm{d}}+\alpha_{\mathrm{q}}=17^{\circ}+29.3^{\circ}=46.3^{\circ}$ became much greater. Consequently a wingtip vortex (WTV) and leading edge vortex (LEV) was generated from the outboard wing. The vortex below the wing generated in earlier time-a was partly captured, and partly shed off the TE as shown in Fig.14(b). In this flow and AoA condition, the FWR produced a relatively large $C_{\mathrm{L}}=1.5$, but negative $C_{\mathrm{M}}=-0.9$ as shown in Fig. 11 .

At time-c, the wing moved into the $2^{\text {nd }}$ half of down-stroke in the mid of the $3^{\text {rd }}$ quarter cycle with geometric AoA $=-5^{\circ}$ as shown in Fig.11. The resulting equivalent angle was largely decreased from earlier time-b value, but remained a large value $\alpha_{e}=\alpha_{\mathrm{u}}+\alpha_{\mathrm{q}}=-5^{\circ}+29.3^{\circ}=24.3^{\circ}$. Consequently the connected LEV and WTV kept growing and maintaining touch to the LE as shown in Fig.14(c). The strong LEV and large $\alpha_{\mathrm{e}}$ led the lift coefficient to nearly the maximum value $C_{\mathrm{L}}=4.0$. Due to the large $C_{\mathrm{L}}$ and negative AoA=-5 $5^{\circ}$, the resulting $C_{\mathrm{M}}$ also reached nearly maximum positive $C_{\mathrm{M}}=1.0$ in this cycle as shown in Fig.11.

At time-d, the wing completed the transition from down-stroke to the first half of up-stroke in the mid of $4^{\text {th }}$ quarter cycle. Due to significant aeroelastic effect in accelerating motion, the geometric AoA increased to $39^{\circ}$ towards the maximum value of the cycle as shown in Fig. 11 . The equivalent AoA became $\alpha_{e}=\alpha_{\mathrm{u}}+\alpha_{\mathrm{q}}=39^{\circ}-29.3^{\circ}=9.7^{\circ}$. The resulting LEV generated at earlier time-c moved down-stream and shed off the TE as shown in Fig.14(d). Due to the large AoA but reduced LEV, the resulting $C_{\mathrm{L}}=0.5$ was reduced from previous value and the $C_{\mathrm{M}}=-0.9$ became negative as shown in Fig. 11 .

At time-e, the wing moved to the mid of up-stroke where the flapping velocity was close to maximum with the $\mathrm{AoA}=35^{\circ}$ reduced from the earlier peak angle $42^{\circ}$ as shown in Fig. 11 . This resulted in an equivalent $\alpha_{e}=\alpha_{\mathrm{u}}+\alpha_{\mathrm{q}}=35^{\circ}-29.3^{\circ}=5.7^{\circ}$. Similar to the time-a, the clean upper surface and vortex below the wing surface resulted in a negative $C_{\mathrm{L}}=-1.2$. Due to the negative $C_{\mathrm{L}}$ and large $\mathrm{AoA}=35^{\circ}$ however, a positive $C_{\mathrm{M}}=1.2$ was produced just before completing the flapping cycle as shown in Fig.11.

\section{Flight test and simulation of the flyable FWR model}

\subsection{The FWR model flight test}

Based on the above results, it was predicted that the FWR can produce sufficient lift force to overcome the model weight $(2.6 \mathrm{gm})$ for the input power below $0.28 \mathrm{~W}(4 \mathrm{v})$. The FWR model was then removed from the load cell and set on a base rail by placing its long legs into a couple of holes as shown in Fig. 15(a). The electric power was supplied to the motor through fine wires, which were kept loosely connected to the FWR motor to ensure little interference with the flying model during flight. When the power of $0.26 \mathrm{~W}$ (about $3.8 \mathrm{v}$ ) was supplied, the FWR model achieved a successful vertical take-off as shown in Fig. 15(b). The measured FWR flapping frequency, rotation speed and $S t$ are $21 \mathrm{~Hz}, 7.2 \mathrm{r} / \mathrm{s}$ and 0.31 respectively. The flyable model demonstrated its self-stabilized flight without control, which is one of the FWR features. By tuning the input power, the FWR model also demonstrated its capability of hovering within a small highlighted space as shown in the Fig.16. 


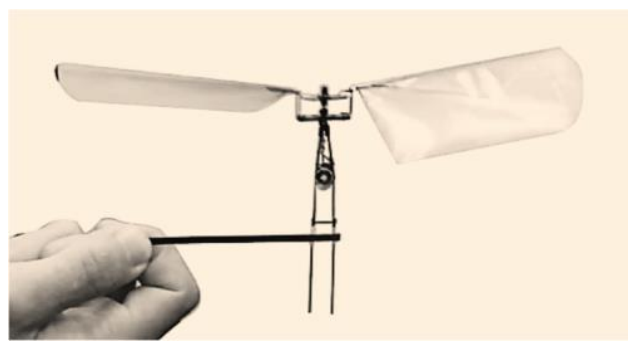

(a)

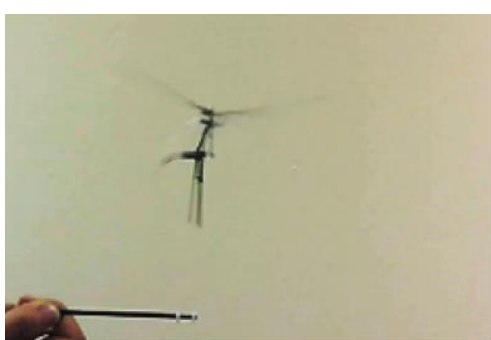

(b)

Figure 15. The FWR model (a) set on a flight launch base (b) vertical take-off
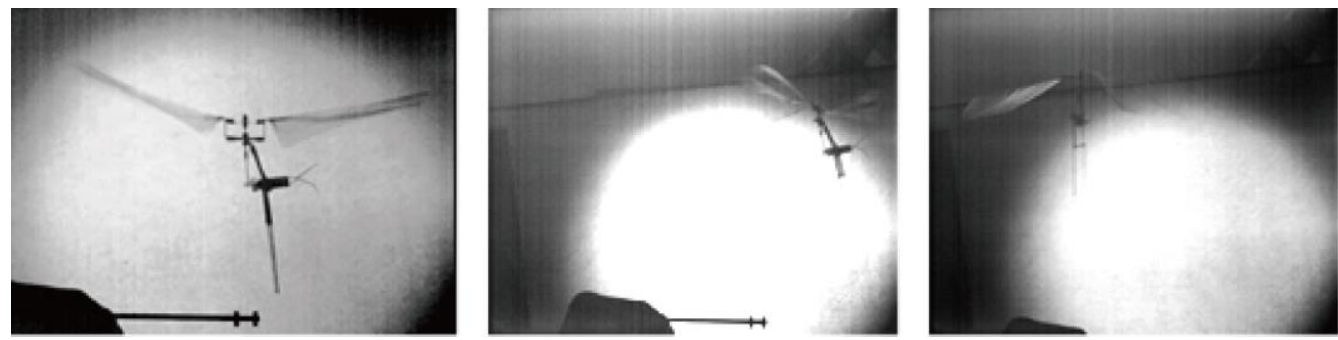

Figure 16. The FWR model hovering within a small highlighted space

In theory, the FWR is statically stable in the $x-y$ plane of the inertia frame system as defined in Fig.1 and free-of-rotation body about the y-axis. This is because no actuation torque is required for the self-propelling wing rotation as one of the FWR key features. In practice however, the FWR model body was swing and spinning although at very low frequency and dynamically stable in both the $x-y$ and $x-z$ plane as observed during the flight test. Throughout the flight test, the fine wires are loosely connected to the motor to prevent external interference to the FWR model movement. The main reason for the model swing is because of the torque about the horizontal z-axis produced by the motor instead of a desirable torque-free linear motor. The body spin is due to the existing friction in the bearings. The additional rotational mass moment of inertia due to the eccentric CG of the FWR model reduces the body spinning frequency.

\subsection{The FWR model flight simulation}

In order to reveal the characteristics of the flight dynamics of the FWR model, a numerical simulation analysis was performed using the software ADAMS interfacing with the aerodynamic forces calculated using the QS method. The dimensions and weight were measured for each of the 21 individual components of the flyable FWR model including the motor, wing LE beam, mid-beam, skin, shaft, crank, bearing and etc. Based on the data, the FWR multi-body dynamic model and the trajectory simulation of the flapping wingtip including the gravity, inertia and aerodynamic forces and motor torque was created using ADAMS as shown in Fig.17.

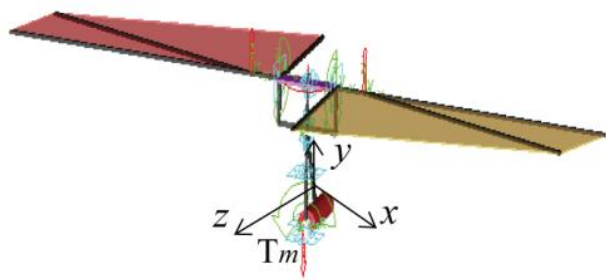

(a)

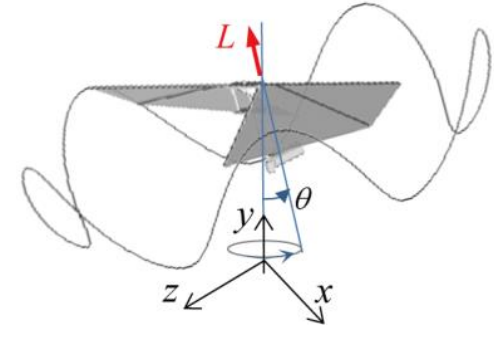

(b)

Figure 17. (a) The flyable FWR model; (b) body and wingtip motion simulation 
The torque $\mathrm{T}_{m}$ produced by the motor as shown in Fig. 17(a) forces the FWR model sways in a small angle $\theta$ and so does the aerodynamic lift force $L$ through the FWR centre line as illustrated in Fig. 17(b). Along with the FWR rotation, the FWR model spins at the same rotation speed. When the bearing frication in the ADAMS model is ignored, the dynamic motion at the motor CG was simulated in the X-z plane as shown in Fig. 18(a) and (b). The results demonstrate that the model spins at oscillation frequency about $4 \mathrm{~Hz}$, which agrees with the FWR rotation speed $4 \mathrm{r} / \mathrm{s}$. The eccentric motor offers additional mass moment of inertia to reduce the swing amplitude and stabilise the model. Consequently, the model (CG) moves away from its original position $(x=0, z=3.5 \mathrm{~mm})$ to a new location $(x=4 \mathrm{~mm}$ and $z=-$ $1 \mathrm{~mm}$ ) after $4 s$. In the same time, the FWR model was lifted up to $30 \mathrm{~mm}$ in the vertical $\mathrm{y}$ direction with small oscillating amplitude in the same frequency as the wing flapping frequency $21 \mathrm{~Hz}$ as shown in Fig. 18(c).

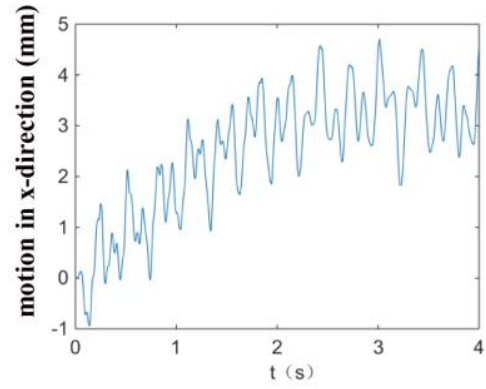

(a)

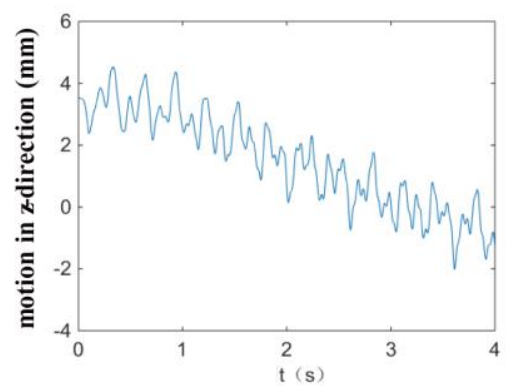

(b)

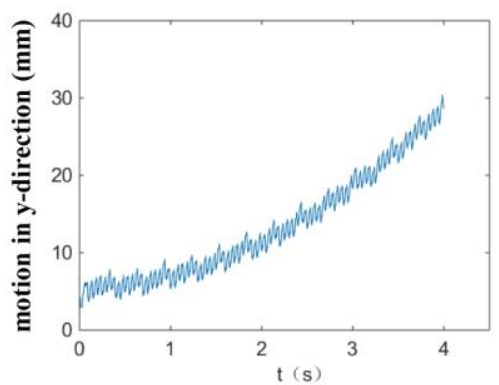

(c)

Figure 18. The motion of the FWR model in (a) $x$; (b) $z$; (c) $y$-direction

When a dynamic friction coefficient 0.1 was set in the bearings of the ADAMS model, the flight motion of the FWR model was also simulated. As shown in Fig. 19(a) and (b), the model swing amplitude remains the same magnitude as the above friction-free results. While the body spinning was initially slower than the friction-free case and gradually increased to $4 \mathrm{~Hz}$ to follow the FWR rotation speed $4 \mathrm{r} / s$ after $2 s$. In the $\mathrm{x}-\mathrm{z}$ plane, the FWR body swings around the original $x=0$ with a small shift in z-direction from $z=-1.5 \mathrm{~mm}$ to $z=0$. In the same time, the FWR model flies up to $30 \mathrm{~mm}$ in vertical direction after $4 \mathrm{~s}$ while oscillating in the flapping frequency $21 \mathrm{~Hz}$ as shown in Fig. 19(c).

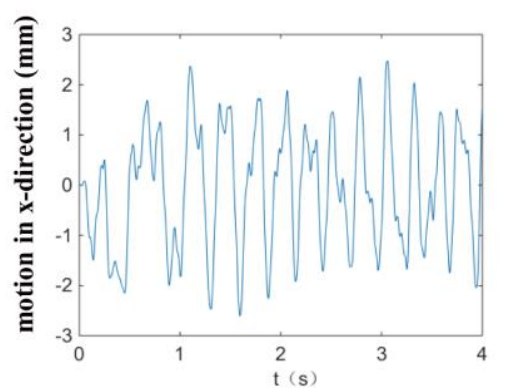

(a)

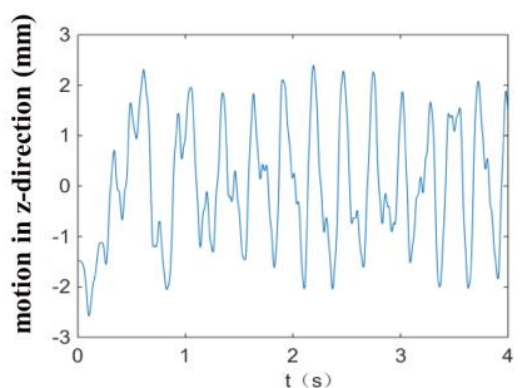

(b)

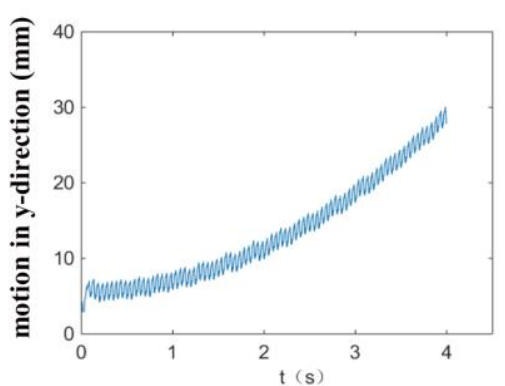

(c)

Figure 19. The motion of the FWR model in (a) $x$, (b) $z$, (c) $y$-direction with bearing friction

The 3D vertical take-off trajectory simulation using ADAMS and the experimental flight dynamic motion in vertical direction measured in the FWR model flight test is shown result Fig.20. 


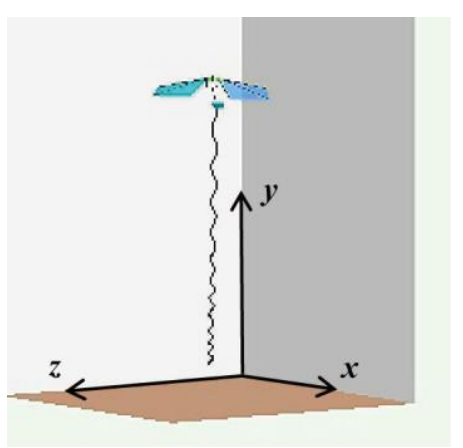

(a)

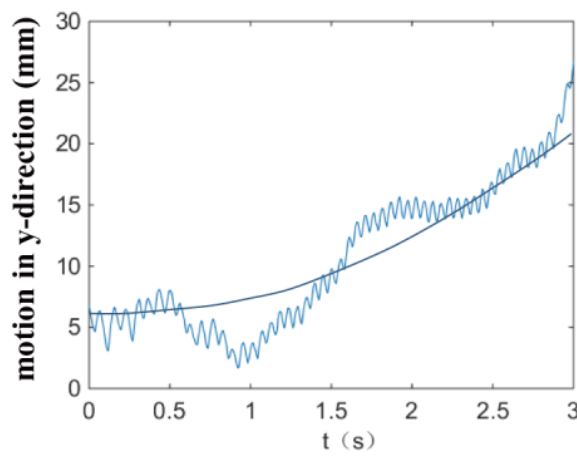

(b)

Figure 20. (a) Vertical take-off simulation; (b) measured flight trajectory of the FWR model

By comparing the results shown in Fig.19 and Fig.20, it is noted that the flight trajectory simulation result agrees with the averaged trace and flight height of the FWR model test. The detailed periodic motion of the FWR model during the flight test is off the trace of the simulation trajectory. This is mainly because the aerodynamic forces used in the ADAMS model were based on an average aerodynamic lift coefficient per flapping cycle by the QS method to represent the unsteady aerodynamic forces in the FWR model flight in real life.

\section{Conclusions}

The bioinspired novel FWR that combines rotary and insect-like flapping kinematics of motion offers an alternative option for high performance MAV. Although systematic studies on the FWR have been performed previously, it is the first time to demonstrate the feasibility of a flyable micro FWR model and perform an extensive study by analytical, numerical and experimental methods. Based on the study, the following conclusions can be drawn.

- A flyable micro FWR model of weight $2.6 \mathrm{gm}$ has been designed and manufactured. This is the first and lightest flyable FWR model developed so far in the research field of bioinspired flapping wings.

- Since a suitable commercial linear actuator or motor was unavailable, an off-the-shelf motor was selected for the FWR model according to the power/weight and gear ratio requirement. The crank-linkage made to transform the motor rotation to linear flapping motion causes not only additional weight and complexity of the mechanism, but also a torque about the horizontal axis of the model. If a suitable linear actuator were available, the problem would have been eliminated to make a simpler and lighter FWR model.

- The elastic plate plays a key role in the lever mechanism of the FWR model. It not only amplifies the flapping angle, but also produces elastic twist angle for the FWR to obtain an optimal kinematics of motion and aeroelastic effect.

- The flyable FWR model provides a desirable base not only for analytical and experimental study in a range of input power, but also a flight demonstrator of the FWR feasibility and advantages.

- The mean aerodynamic lift coefficients and average forces by the analytical method have excellent agreement with the experimental results $(<3.4 \%$ difference $)$ within the specified normal operation range of power for the motor $(3 \sim 3.5 \mathrm{~V})$.

- The resulting kinematics of motion and passive rotation of the FWR model lead to an optimal propelling efficiency in terms of $\mathrm{St}=0.25 \sim 0.4$ for the whole range of input power $(0.25 \sim 4.5 \mathrm{v})$.

- The simulation of instantaneous flow field by the CFD method based on the measured FWR motion over a flapping cycle reveals that the resulting LEV and $C_{\mathrm{L}}$ depend upon the quasi-steady AoA; the $C_{\mathrm{M}}$ depends upon the $C_{\mathrm{L}}$ and geometric AoA. The maximum 
$C_{\mathrm{L}}=4.0$ and simultaneous $C_{\mathrm{M}}=1.0$ due to the large $\alpha=17.3^{\circ}$ and negative $\mathrm{AoA}=-5^{\circ}$ at the time-C show an ideal result of the FWR flapping status.

- A successful VTOL and short hovering flight test has been performed. This is the first time in history and a breakthrough achievement to prove the capability and stability of the novel FWR flight. This extended experiment demonstrates the unique features and advantages proposed in the FWR concept and also provides useful data for analytical model validation.

- The ADAMS model built based on the measured dimensions and mass data of the individual components provides an accurate representative of the flyable micro FWR. The flight simulation results give an accurate prediction of the FWR body motion and flight trajectory. The swing and spinning motion of the FWR body during flight reflects the influence of the motor torque and eccentric $\mathrm{CG}$ on the flight behaviour of this particular FWR model.

\section{Acknowledgments}

The authors acknowledge the financial support made by Chinese SAFEA to the high-end foreign expert research program (GDW20163500194); fundamental research funds of Shandong University (2015JC026).

\section{References}

[1] R. Żbikowski, Fly like a Fly, IEEE Spectrum 42 (2005) 46-51.

[2] D. Lentink, Biomimetics: Flying like a fly, Nature. 498 (2013) 306-307

[3] W. Shyy, M. Berg, D. Ljungqvist, Flapping and flexible wings for biological and micro air vehicles, Progress in Aerospace Science 35 (1999) 455-505.

[4] C.P. Ellington, The novel aerodynamics of insect flight: applications to micro-air vehicles, Journal of Experimental Biology 202 (1999) 3439-3448.

[5] C.P. Ellington, The Aerodynamics of Hovering Insect Flight .4. Aerodynamic Mechanisms, Philos. Trans. R. Soc. London Ser. B-Biological Sci. 305 (1984) 79-86

[6] C.P. Ellington, C. van den Berg, A.P. Willmott, A.L.R. Thomas, Leading-edge vortices in insect flight, Nature. 384 (1996) 626-630

[7] M.H. Dickinson, K. Götz, Unsteady aerodynamic performance of model wings at low reynolds numbers, J. Exp. Biol. 174 (1993) 45-64.

[8] M.H. Dickinson, F.O. Lehmann, S.P. Sane, Wing rotation and the aerodynamic basis of insect flight, Science (80-. ). 284 (1999) 1954-1960

[9] T.T. Lim, C.J. Teo, K.B. Lua, K.S. Yeo, On the prolong attachment og leading edge vortex on a flapping wing, Mod. Phys. Lett. B. 23 (2009) 357-360.

[10] D. Lentink, M.H. Dickinson, Rotational accelerations stabilize leading edge vortices on revolving fly wings, J. Exp. Biol. 212 (2009) 2705-2719.

[11] Y.J. Lee, K.B. Lua, T.T. Lim, Aspect ratio effects on revolving wings with Rossby number consideration., Bioinspir. Biomim. 11 (2016) 56013. doi:10.1088/1748-3190/11/5/056013.

[12] J.R. Usherwood, C.P. Ellington, The aerodynamics of revolving wings - I. Model hawkmoth wings, J. Exp. Biol. 205 (2002) 1547-1564.

[13] M. Sun, J.H. Wu, Aerodynamic force generation and power requirements in forward flight in a fruit fly with modeled wing motion, J. Exp. Biol. 206 (2003) 3065-3083.

[14]Z.J. Wang, J.M. Birch, M.H. Dickinson, Unsteady forces and flows in low Reynolds number hovering flight: two-dimensional computations vs robotic wing experiments, J. Exp. Biol. 207 (2004) 449-460.

[15] J.H. Wu, M. Sun, Unsteady aerodynamic forces of a flapping wing, J. Exp. Biol. 207 (2004) 1137-1150.

[16]D. Lentink, S.R. Jongerius, N.L. Bradshaw, The Scalable Design of Flapping Micro-Air Vehicles 
Inspired by Insect Flight, Fly. Insects Robot. (2009) 185-205.

[17] S. Guo, D. Li, Z. Huang, A smart material aeroelastic flapping wing micro rotorcraft, in: Int. Forum Aeroelasticity Struct. Dyn., 2009. Seattle, Washington, IFASD-2009-163.

[18] S. Guo, D. Li, J. Wu, Theoretical and experimental study of a piezoelectric flapping wing rotor for micro aerial vehicle, Aerosp. Sci. Technol. 23 (2012) 429-438.

[19] C. Zhou, J. Wu, S. Guo, D. Li, Experimental study on the lift generated by a flapping rotary wing applied in a micro air vehicle, Proc. Inst. Mech. Eng. Part G-J. Aerosp. Eng. 228 (2014) 20832093

[20] J. Wu, D. Wang, Y. Zhang, Aerodynamic Analysis of a Flapping Rotary Wing at a Low Reynolds Number, AIAA J. 53 (2015) 2951-2966.

[21]H. Li, S. Guo, YL. Zhang, C Zhou, JH. Wu, Unsteady aerodynamic and optimal kinematic analysis of a micro flapping wing rotor, Aerospace Science and Technology, Vol.63, (2017) 167178

[22] Wu Jianghao, Zhou Chao, Zhang Yanlai, Aerodynamic power efficiency comparison of various micro-air-vehicle layouts in hovering flight, AIAA Journal, Vol 55, No.4 (2017), 1265-1278.

[23] S.P. Sane, M.H. Dickinson, The control of flight force by a flapping wing: Lift and drag production, J. Exp. Biol. 204 (2001) 2607-2626.

[24] Z.J. Wang, J.M. Birch, M.H. Dickinson, Unsteady forces and flows in low Reynolds number hovering flight: two-dimensional computations vs robotic wing experiments, J. Exp. Biol. 207 (2004) 449-460.

[25] J.P. Whitney, R.J. Wood, Aeromechanics of passive rotation in flapping flight, J. Fluid Mech. 660 (2010) 197-220.

[26] A. Andersen, U. Pesavento, Z.J. Wang, Unsteady aerodynamics of fluttering and tumbling plates, J. Fluid Mech. 541 (2005) 65

[27] L.I. Sedov, Two-dimensional problems of hydrodynamics and aerodynamics, New York, NY: Interscience Publishers. (1980) 20-30.

[28] G. Taylor, R. Nudds, and A. Thomas, Nature, 425, 707-711 (2003).

[29] J. Young, S.M. Walker, R.J. Bomphrey, G.K. Taylor, A.L.R. Thomas, Details of insect wing design and deformation enhance aerodynamic function and flight efficiency, Science (80-. ). 325 (2009) 1549-1552. 
2018-06-06

\section{Analysis and experiment of a} bio-inspired flyable micro flapping wing rotor

Guo, S.

Elsevier

Guo S, Li H, Zhou C, et al, Analysis and experiment of a bio-inspired flyable micro flapping wing rotor. Aerospace Science and Technology, Volume 79, Issue August, 2018, pp. 506-517

https://doi.org/10.1016/j.ast.2018.06.009

Downloaded from Cranfield Library Services E-Repository 